\title{
An improved technique for aortic anastomosis: Graft telescopic inversion
}

\author{
Bartosz Rylski, MD, Matthias Siepe, MD, Joachim Schoellhorn, MD, and Friedhelm Beyersdorf, MD, PhD, \\ Freiburg, Germany
}

Different anastomotic options have been described for reconstructive prosthetic techniques on the aorta. ${ }^{1-4}$ Problems associated with aortic surgery are usually caused by the degeneration of aortic tissues because of dissection, aneurysm, or atherosclerosis. Relevant complications include anastomotic side bleeding, chronic false aneurysms, and dissection of the remaining aorta. We have developed an anastomotic technique that involves inverting the graft at the proximal and distal ends and reinforcing with a felt strip on the external border of the aortic wall.

\section{CLINICAL SUMMARY}

Aortic anastomosis was performed by using the method described below. The ascending aorta was crossclamped proximal to the innominate artery during an elective surgical procedure. For dissection, the axillary artery was cannulated and the ascending aorta was replaced with antegrade selected cerebral perfusion with occlusion of the brachiocephalic trunk. After initiating retrograde blood cardioplegia, the diseased ascending aorta was resected. A 3-0 polypropylene suture was used for the proximal and distal ascending aortic graft anastomoses (Johnson \& Johnson International, St Stevens Woluwe, Belgium).

First, the aortic wall was reinforced with an extraluminal circumferential felt strip attached with a separate suture. A Dacron (Vascutek, Inchinnan, Scotland) tube graft of appropriate size was cut obliquely and held opposite to the transected aorta, maintaining a distance of about $3 \mathrm{~cm}$ and providing excellent exposure of the dorsal aortic wall. The running suture was started with the graft. The anastomosis was made with a continuous suture, the needle of which went through the graft (out-in), through the aorta (in-out), and again through the aorta (out-in) to invaginate 3 to 5 $\mathrm{mm}$ of the graft into the aorta. This technique therefore requires 3 stitches (aorta twice) for each turn. When the dorsal

From the University Cardiovascular Center, Freiburg-Bad Krozingen, Freiburg, Germany.

Disclosures: None.

Received for publication Feb 20, 2010; revisions received April 1, 2010; accepted for publication April 25, 2010; available ahead of print June 28, 2010.

Address for reprints: Bartosz Rylski, MD, University Cardiovascular Center FreiburgBad Krozingen, Hugstetter Str. 55, 79106 Freiburg, Germany (E-mail: bartosz. rylski@uniklinik-freiburg.de).

J Thorac Cardiovasc Surg 2010;140:934-5

$0022-5223 / \$ 36.00$

Copyright (c) 2010 by The American Association for Thoracic Surgery doi: $10.1016 /$ j.jtcvs. 2010.04 .024 part of the anastomosis was completed, the suture was pulled tight, we inverted the end of the graft into the aorta's lumen, and the suture was continued in the same manner as described. The running suture and the suture for the external felt were carefully retightened and knotted at the end of the anastomosis to avoid an anastomotic stenosis (Figure 1).

Since 2008, 21 consecutive patients have undergone safe elective or emergency surgical procedures for aortic aneurysm or dissection by one surgeon using this technique. The anastomosis required about $40 \%$ more time than the simple "over-and-over" technique. All patients underwent ascending aortic replacement, with 7 of them undergoing the David procedure. There have been no reoperations for bleeding or false aneurysm and no deaths.

\section{DISCUSSION}

One of the crucial aspects of surgical repair of a diseased ascending aorta is to achieve anastomotic hemostasis. An aortic wall affected by aneurysm or dissection is obviously vulnerable, necessitating a particularly secure anastomosis. With this method, the aortic wall is supported on the outside by a felt strip and on the inside by inverting the prosthesis, mimicking the effect of a double-felt anastomosis. The degree of security and tightness resembles that of a double-felt anastomosis; however, this technique requires no foreign material, which is in contrast to a technique with inside felt that might elicit embolic events. Inverted Dacron aortic anastomoses held sutures well and acted as aortic wall reinforcement, providing complete hemostasis. Moreover, it is an inexpensive, quick, and easily learned technique applicable during elective and emergency surgical interventions. It is equally adaptable for distal and proximal anastomoses and does not produce a ridge projecting into the blood stream, as made evident in routine, postoperative, high-resolution computed tomographic scans. We identified no false aneurysms or other anastomotic problems during the late follow-up periods for most patients. These results are limited by the small sample size.

Various anastomotic options have been described for reconstructive prosthetic techniques on the aorta. The anastomosis described is superior to use of interrupted sutures, ${ }^{1,3}$ which requires more time for their placement. When compared with the "turn-up" method, ${ }^{1,2}$ there is no ridge in the bloodstream in our technique. The inversion of the graft in the aorta is secured by 2 aortic stitches for each turn, making this technique particularly safe and reducing significantly 

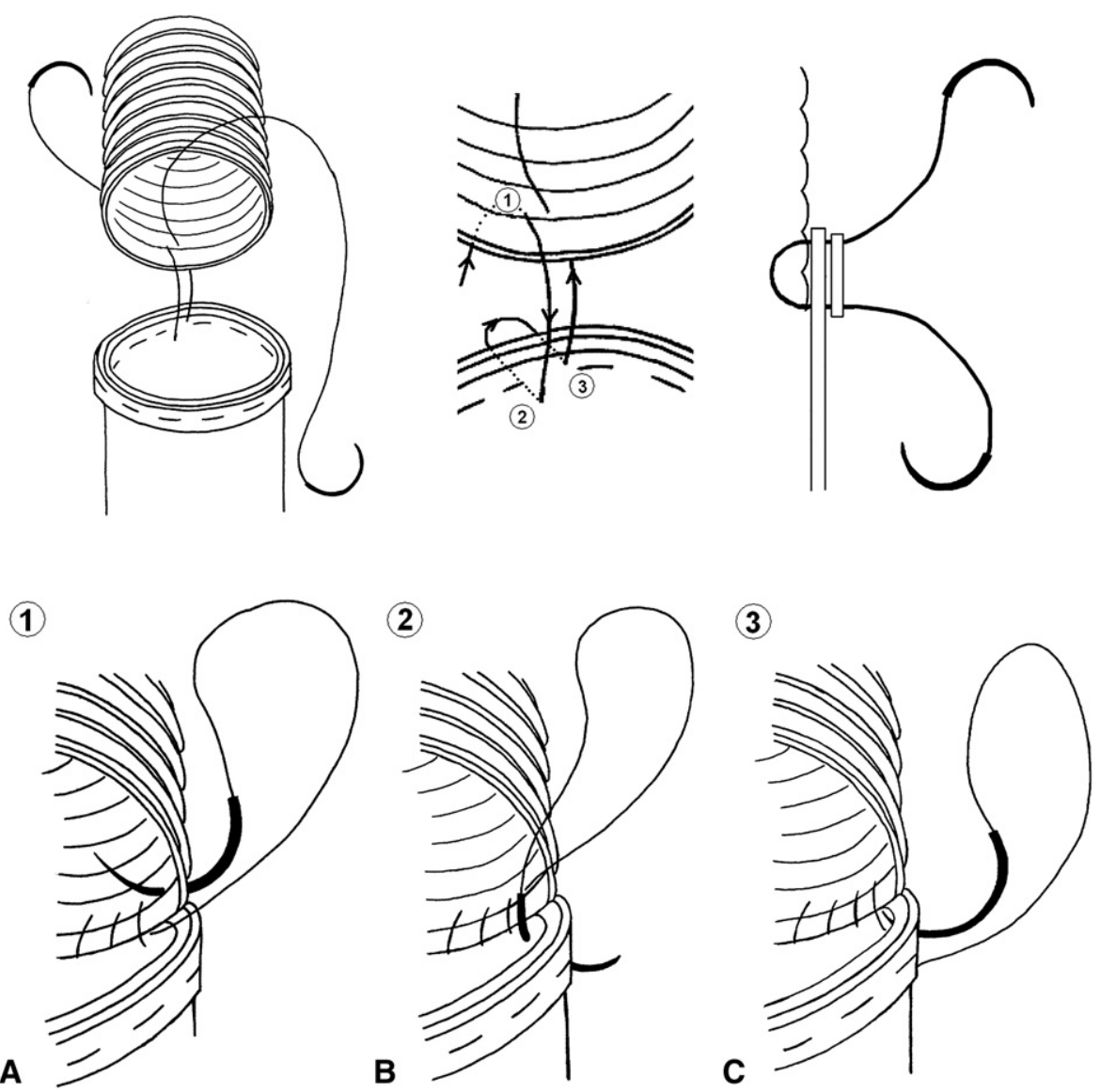

FIGURE 1. A technique for end-to-end inverted Dacron aortic anastomosis. A, The aortic wall was reinforced with an extraluminal felt strip attached with a separate suture. A tube graft was held opposite to the transected aorta, maintaining a distance of about $3 \mathrm{~cm}$ for excellent exposure of the ventral aortic wall. $\mathrm{B}$, The anastomosis was performed with a continuous suture, the needle of which went through the graft (out-in; 1), through the aorta (in-out; 2), and again through the aorta (out-in; 3), invaginating 3 to $5 \mathrm{~mm}$ of graft into the aorta. C, With this method, the aortic wall is supported on the outside by a felt strip and on the inside by inverting the prosthesis, mimicking the effect of a double-felt anastomosis.

the need for hemostatic stitches after completion of the anastomosis. We do not recommend this technique for all aortic anastomoses in any given circumstance. However, we believe that it is a meaningful add-on for our surgical armamentarium that one can easily try first in a straightforward case or on an animal's aorta ex vivo.

We have used this technique solely in ascending aortic surgery. However, it can be easily implemented in descending aortic surgery or arch replacement as well.

\section{References}

1. Tamura N, Komiya T, Sakaguchi G, Kobayashi T. "Turn-up" anastomotic technique for acute aortic dissection. Eur J Cardiothorac Surg. 2007;31:548-9.

2. Rignano A, Keller GC, Carmo M, Anguissola GB, Settembrini PG. A new approach for proximal anastomosis in type "A" acute aortic dissection: prosthesis eversion. Ann Thorac Surg. 2003;76:949-51.

3. Farivar RS, Cohn LH. An improved hemostatic aortic anastomotic technique for nondissected aortic aneurysms. J Thorac Cardiovasc Surg. 2009;137:1570-1.

4. Tanaka K, Morioka K, Li W, Yamada N, Takamori A, Handa M, et al. Adventitial inversion technique without the aid of biologic glue or Teflon buttress for acute type A aortic dissection. Eur J Cardiothorac Surg. 2005;28:864-9. 